\title{
Management of Visceral Artery Pseudoaneurysms by Combined Technique of Percutaneous Thrombin Injection and Endovascular Coiling
}

\author{
Kalpana Sivalingam ${ }^{1}$ Karthik Palanisamy ${ }^{1}$ Amalan Ignatius ${ }^{1}$ \\ ${ }^{1}$ Barnard Institute of Radiology, Madras Medical College, Chennai, \\ Tamil Nadu, India \\ Address for correspondence Kalpana Sivalingam, DMRD, MDRD, \\ DNB, PhD, Barnard Institute of Radiology, Madras Medical College, \\ Chennai, Tamilnadu, India (e-mail: kapudr@gmail.com).
}

J Clin Interv Radiol ISVIR 2021;5:172-176.

\begin{abstract}
Purpose To describe our initial experience with percutaneous thrombin injection combined with endovascular coiling of high-flow visceral artery pseudoaneurysms. Materials and Methods Institutional review board approval was obtained. Between January 2019 and February 2020, 21 patients with nonvariceal upper gastrointestinal (GI) bleed underwent multidetector CT angiography. Four patients with acute pancreatitis and large pseudoaneurysms were selected for the combined technique. Human thrombin reconstituted with calcium chloride was injected percutaneously. Partially thrombosed aneurysms were treated up to three times. Simultaneously or within 15 days, coil embolization of the neck or patent part of pseudoaneurysm was performed. Patients were followed up to 1 month postprocedure.

Results The size of the pseudoaneurysms ranged from $3 \mathrm{~cm}$ to $6 \mathrm{~cm}$. All were accessed percutaneously by $22 \mathrm{G}$ Chiba needle under ultrasound guidance and 500 to 1500 units of thrombin was injected in one to three attempts. Subsequently, endovascular coiling of the neck of the pseudoaneurysm or of the feeding artery was performed. Technical success with cessation of flow was achieved in all four patients.

Keywords

- pseudoaneurysms

- thrombin

- coiling

One patient developed obstructive jaundice postprocedure and another continued to have GI bleeding despite thrombin injection.

Conclusion Percutaneous thrombin injection is an easy to use and effective tool for treating visceral artery pseudoaneurysms and can be combined with endovascular coiling for successful occlusion of large pseudoaneurysms.
\end{abstract}

\section{Introduction}

Visceral arterial pseudoaneurysms, although rare, are a significant source of gastrointestinal (GI) bleeding. They are preferably managed by transcatheter techniques such as coiling, glue injection, or particle embolization. Percutaneous thrombin injection, directly into pseudoaneurysms, was initially attempted successfully for iatrogenic femoral arterial pseudoaneurysms and later for treatment of visceral arterial pseudoaneurysms that failed endovascular therapy. We describe our initial experience with the combined technique of percutaneous thrombin injection and endovascular coiling in the treatment of visceral arterial pseudoaneurysms in four patients. published online June 1, 2021
DOI https://doi.org/

$10.1055 / \mathrm{s}-0041-1728977$

ISSN 2457-0214 (c) 2021. Indian Society of Vascular and Interventional Radiology. This is an open access article published by Thieme under the terms of the Creative Commons Attribution-NonDerivative-NonCommercial-License, permitting copying and reproduction so long as the original work is given appropriate credit. Contents may not be used for commercial purposes, or adapted, remixed, transformed or built upon. (https://creativecommons.org/licenses/by-nc-nd/4.0/).

Thieme Medical and Scientific Publishers Pvt. Ltd. A-12, 2nd Floor, Sector 2, Noida-201301 UP, India 


\section{Materials and Methods}

Institutional review board approved this prospective study. Between January 2019 to February 2020, 21 patients with nonvariceal upper GI bleed underwent multidetector CT angiography (16 slice Siemens Somaton). Sixteen patients had pseudoaneurysms due to acute pancreatitis. Four patients with large pseudoaneurysms, which could be accessed percutaneously, were selected for combined technique. Smaller aneurysms inaccessible by percutaneous method were managed by the endovascular approach. Percutaneous thrombin was injected into the pseudoaneurysm in all four patients. We used human thrombin (either Floseal or Tisseal) reconstituted with calcium chloride such that each $\mathrm{mL}$ of the solution contained 500 units of thrombin. Under ultrasound guidance, 1000 units of reconstituted thrombin was loaded into the syringe and slowly injected with ultrasound monitoring into the pseudoaneurysm via a 22 G Chiba needle. Instant thrombosis of the pseudoaneurysm was depicted as echogenicity within the pseudoaneurysm. The needle tip was redirected to portions that were patent and thrombin was reinjected in an attempt to completely occlude the pseudoaneurysm. Partially thrombosed portions were subjected to maximum of three repeat injections. Either simultaneously or within 30 days, angiographic access was obtained via right femoral route, and selective catheterization of the feeding artery was performed using Cobra/Sims catheter for Celiac access and Progreat (Terumo, Japan) microcatheter for distal selective access. Coil embolization of the neck or patent part of pseudoaneurysm was performed. Follow-up Doppler on day three and either Doppler or contrast-enhanced CT after 1 month was performed to check for residual flow.

\section{Individual Case Reports}

\section{Case Report 1}

A 42-year-old male with acute pancreatitis presented with complaints of abdominal pain and repeated episodes of hematemesis. Serum amylase and lipase were grossly elevated. CT scan revealed multiple pseudocysts, pancreatic calcification, and $3.2 \times 4.8 \mathrm{~cm}$ aneurysm from distal splenic artery ( - Fig. 1A). Colur doppler confirmed aneurysm near splenic hilum (-Fig. 1B). Through percutaneous access via 22G Chiba needle under ultrasound guidance thrombin was injected into the pseudoaneurysm and resulted in partial thrombosis. The needle was redirected to the residual patent portion and the remaining thrombin solution was injected with partial success. A total of 1000 units of thrombin was injected. Third day, Doppler revealed residual patency near neck of the aneurysm ( $\mathbf{- F i g}$. 1C). The aneurysm failed to thrombose spontaneously and as Doppler revealed patency near the neck, 500 units of thrombin was injected on day 10 and 15 with failure to occlude aneurysm completely. Doppler study on day 27 revealed patent portion within the pseudoaneurysm, which was inaccessible percutaneously, as
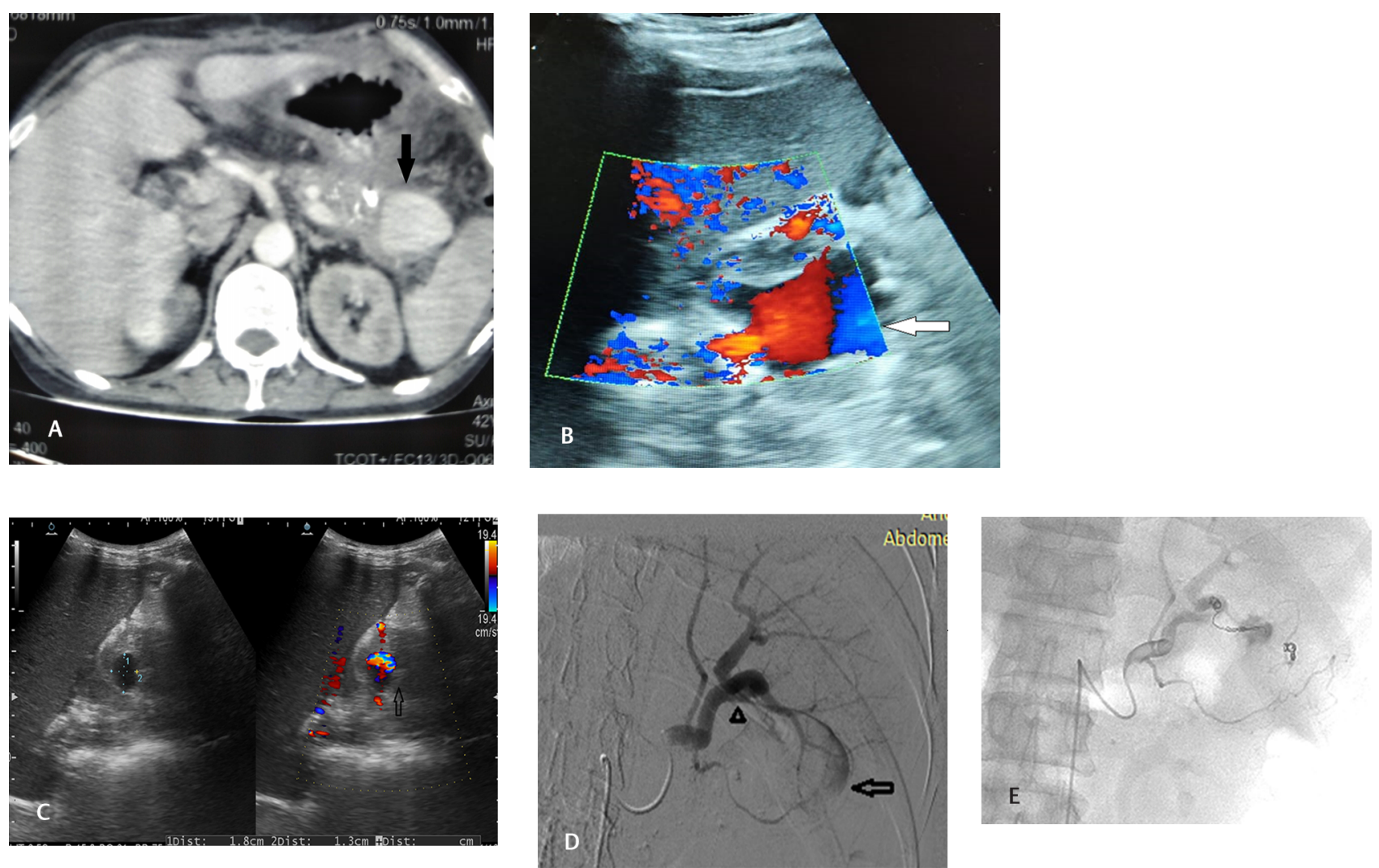

Fig 1 (A) Contrast CT axial section reveals pancreatic calcification and distal splenic artery aneurysm (arrow) in 42-year-old male patient. (B) Color Doppler of large splenic artery aneurysm (arrow) of same patient. (C) Follow-up Doppler on 3rd day reveals partial recanalization (arrow) near neck of pseudoaneurysm. (D) Digital subtraction angiography (DSA) of splenic artery shows tear (arrowhead) in distal splenic artery with partial filling of large pseudoaneurysm (arrow). (E) Postcoiling angiogram shows no flow into aneurysm. 
it was posterior to the spleen. History of melena prompted angiographic embolization of residual patent portion. The pseudoaneurysm was formed by a tear in the wall of splenic artery (-Fig. 1D). The neck was then occluded by $3 \mathrm{~mm}$ and $6 \mathrm{~mm}$ Nester coils via endovascular route. Check angiogram revealed complete occlusion of the pseudoaneurysm $(\boldsymbol{- F i g}$. 1E), and the patient was discharged with cessation of GI bleed after 3 days. Follow-up Doppler after 4 weeks revealed complete occlusion of the pseudoaneurysm.

\section{Case Report 2}

A 38-year-old male presented with severe back pain and history of hematemesis. Laboratory values demonstrated elevation of serum amylase and lipase. Contrast-enhanced CT abdomen revealed pancreatitis with pseudocysts, ascites, and $2 \times 2.4 \mathrm{~cm}$ aneurysm from gastroduodenal artery encased by pancreatic collection ( - Fig. 2A). On angiogram, the common hepatic artery was thinned and draped over the pseudocyst with distal flow into the proper hepatic artery (-Fig. 2B). The large pseudoaneurysm was seen arising within $2 \mathrm{~cm}$ of origin of the gastroduodenal artery, with distal flow into the right gastroepiploic artery. Via percutaneous route, a mixture of 1000 units of thrombin and $2 \mathrm{~mL}$ of calcium chloride (TISSEAL) was injected with instantaneous thrombosis (-Fig. 2C).

The fast flow in the neck was persistent and was occluded simultaneously through an endovascular approach, using
$6 \mathrm{~mm}$ and $5 \mathrm{~mm}$ Nester coils. Postprocedure check angiogram showed complete cessation of flow into pseudoaneurysm ( - Fig. 2D). However, obstructive jaundice developed, and $\mathrm{CT}$ a week after thrombin injection revealed mild biliary obstruction due to extraneous pressure by the hyperdense, calcified, thrombosed aneurysm (-Fig. 2E), which resolved with conservative treatment. The thrombosed aneurysm was hyperdense on plain CT due to the calcium chloride. Follow-up Doppler revealed complete occlusion of the pseudoaneurysm.

\section{Case Report 3}

A 43-year-old man with history of alcoholism presented with upper abdominal pain. Routine blood investigations were normal. CT angiogram showed a wide mouth saccular aneurysm (-Fig. $\mathbf{3 A}$ ) of $2.8 \times 2.7 \times 3.2 \mathrm{~cm}$ arising from the inferior aspect of midsplenic artery, with an adjacent haematoma of $5.8 \times 4 \mathrm{~cm}$ indenting body and tail of the pancreas. Selective splenic arteriography revealed a large saccular aneurysm from midsplenic artery. With angiographic control, 1000 units of reconstituted thrombin was injected percutaneously into the aneurysm. The aneurysm thrombosed in a few seconds after injection. Two $3 \mathrm{~mm}$ coils and one $6 \mathrm{~mm}$ coil were placed across neck of aneurysm to prevent further recanalization. A Doppler study 3 days and a month after the procedure revealed complete occlusion of the pseudoaneurysm (-Fig. 3B).
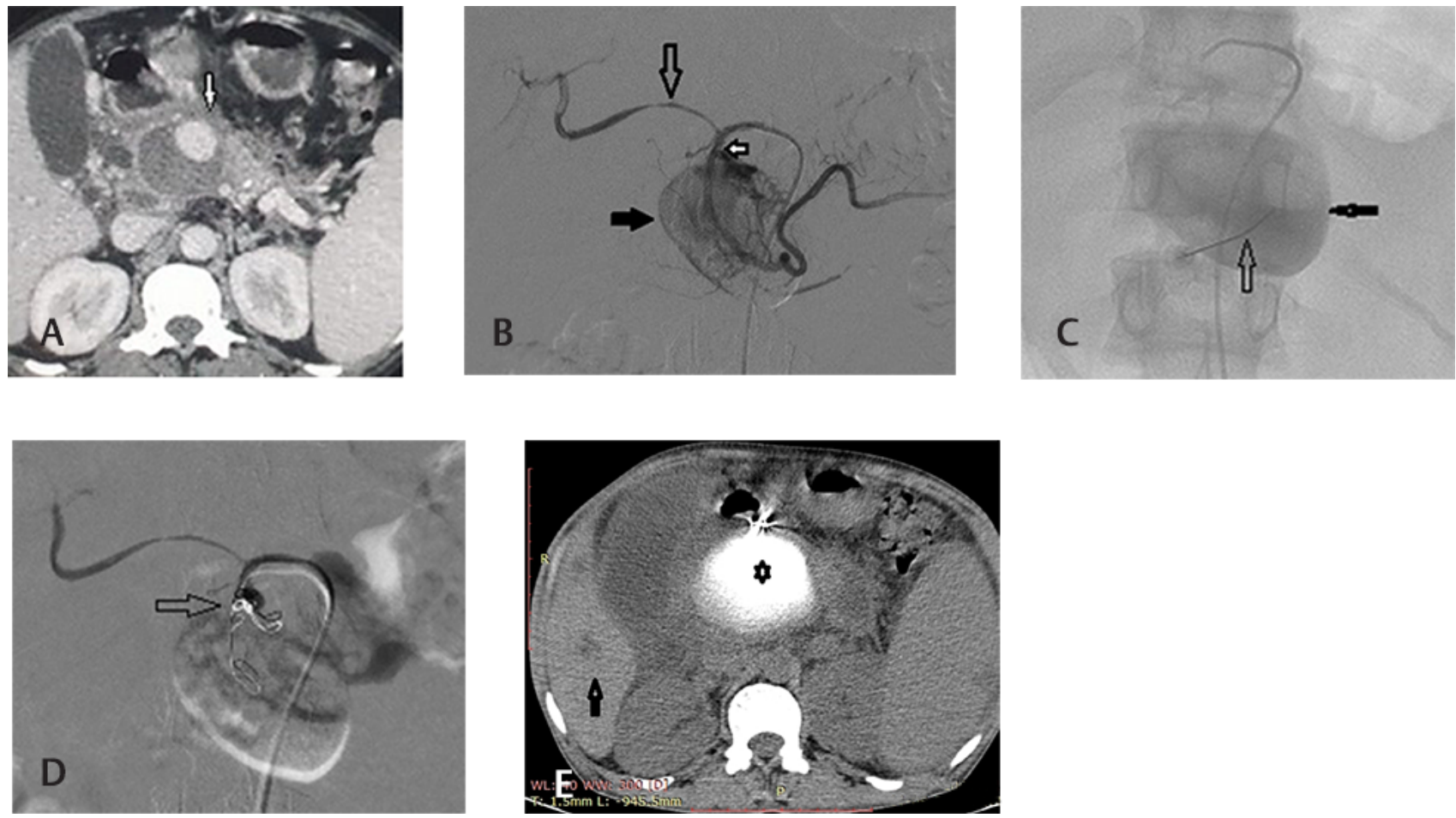

Fig. 2 (A) Contrast CT abdomen depicting large partially thrombosed pseudoaneurysm (arrow) in patient 2. (B) Hepatic artery digital subtraction angiography (DSA) showing thinned and stretched proper hepatic artery (long arrow) over pseudoaneurysm (black arrow) arising from gastroduodenal artery (white solid arrow). (C) Angiogram image showing percutaneous Chiba needle (arrow) for thrombin injection into aneurysm filled with contrast. (block arrow). (D) Check angiogram postcoiling (arrow) and thrombin injection with no flow into pseudoaneurysm. (E) Plain CT abdomen with hyperdense calcified aneurysm (star) causing intrahepatic biliary dilatation (arrow). 


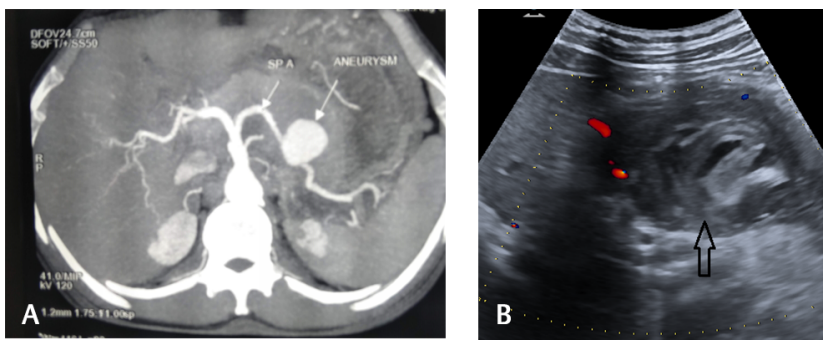

Fig. 3 (A) Maximum intensity projection in patient 4 reveals pseudoaneurysm from splenic artery. (B) Follow-up USG abdomen shows mixed echogenic thrombosed aneurysm (arrow) post thrombin injection.

\section{Case Report 4}

A 36-year-old male patient with pancreatitis and melena was found to have a gastroduodenal artery pseudoaneurysm, measuring $4 \times 2.2 \mathrm{~cm}$ on contrast-enhanced CT. With angiographic control and through a percutaneous approach, 1000 units of thrombin was injected with instant thrombosis of the pseudoaneurysm. Simultaneously, a coil was deployed across the neck of the pseudoaneurysm. Doppler evaluation on day 2 and a month postprocedure showed complete thrombosis of the pseudoaneurysm.

\section{Discussion}

Visceral arterial pseudoaneurysms are best tackled by endovascular techniques. Studies comparing outcomes between endovascular and open surgical repair have found a lower 30-day morbidity and mortality for ruptured aneurysms and a protective effect on 2-year mortality by endovascular techniques. ${ }^{1}$

Most pancreatitis-related pseudoaneurysms arise from the splenic artery, gastroduodenal artery, left gastric artery, or hepatic arteries. Current management strategies include endovascular embolization with coils or glue or exclusion of the pseudoaneurysm with a stent graft. Coiling of the aneurysm is feasible in smaller aneurysms. In larger aneurysms, coils are usually placed proximal and distal to the neck of pseudoaneurysm (sandwich technique) to prevent either antegrade or retrograde filling of the aneurysm. Covered stents are suitable for those aneurysms from major arteries that need preservation of flow such as the hepatic artery. Covered stents lack flexibility while delivery and therefore may not be taken through tortuous arterial routes. ${ }^{2}$

Aneurysms arising from major branches are easier to reach angiographically compared with those from smaller side branches. The angiographic approach is hampered by the fact that these vessels are prone to spasm and stenosis following guide wire manipulation. The alternative to failed endovascular approach is percutaneous approach via which thrombin, glue, and coil placement have been attempted previously with good success rate.

The first report on the use of thrombin for percutaneous embolization of aneurysms for both peripheral and visceral arteries was in 1986 by Cope et al. ${ }^{3}$ Since then, it has been used in treatment of visceral aneurysms as a secondary measure when endovascular access was difficult, and where percutaneous access is feasible.

Thrombin splits fibrin from fibrinogen and polymerizes it into fibrin mesh, which stabilizes into an insoluble state by factor XIII and calcium. The commercially used product is human thrombin, which is mixed with calcium chloride solution. After the injection, it acts immediately; the clot is bioabsorbable, and the risk of infection, tissue necrosis, or inflammation is minimized.

Under ultrasound guidance, the pseudoaneurysm and the neck are visualized and 19 to 22 gauge needle is advanced percutaneously under sterile conditions into the sac. The injection is performed slowly and continually. Thrombosis occurs instantaneously around the needle tip and can be viewed as an echogenic area by ultrasound. Injection is continued until the flow stops within aneurysm on ultrasound. The success of clot formation depends on concentration of thrombin and the degree of stasis within the aneurysm. ${ }^{3}$ Hence, thrombin is better suited for slow flow aneurysm with small necks where it cannot be washed away. Even if thrombin and fibrin escape downstream, they are promptly lysed and no inadvertent organ damage occurs. ${ }^{4}$

The tricks and errors we learnt on percutaneous injection of thrombin for high-flow large aneurysms are: 1) Peripherally directed thrombin gets concentrated only at the site of injection and leaves the central part of sac patent. 2) When the needle is directed toward the neck of the sac, the high flow within distributes the thrombin evenly and near total occlusion can be achieved. 3) Despite the near total/total occlusion of sac with thrombin, the inevitable residual area near the neck that remains persistently patent or recanulates in time requires simultaneous endovascular coiling for a complete success. 4) Complications are minimal and if both techniques are done simultaneously, the presence of catheter in the feeding artery during percutaneous thrombin injection decreases the speed of flowing blood into aneurysm and facilitates thrombosis within the aneurysm.

This two-step treatment effectively stops further flow into the pseudoaneurysm and also stops further GI bleed. Studies have demonstrated rebleed with percutaneous thrombin alone. ${ }^{5}$ Previous authors have performed transabdominal coiling along with percutaneous thrombin. ${ }^{6}$ But the endovascular route provides greater control over the site of coil deployment to effectively control further flow into the pseudoaneurysm. Endovascular coil followed by percutaneous thrombin has been used successfully to treat rebleed.?

This combined technique was technically successful in all four patients. Many a time during coiling of large high-flow aneurysms, a greater number of coils are required for dense packing to achieve complete thrombosis. With this combined technique, we were able to limit the number of coils to maximum of five and bring down the cost of procedure.

The main indication for percutaneous thrombin injection in visceral aneurysms is for slow-flowing aneurysms, which have failed endovascular treatment. Second thrombin 
injection in high-flow aneurysms may be used as a temporary measure in areas where endovascular treatment is not available. The combined technique may be useful in larger aneurysms if there is persistent flow into aneurysm postendovascular coiling.

\section{Conclusion}

Thrombin is an easy to use, effective, occlusive agent for visceral artery pseudoaneurysms, and combined with endovascular coiling, it is an effective tool to treat large visceral pseudoaneurysms. The combined technique offers added advantage in achieving instantaneous complete thrombosis in high-flow aneurysms and limits the number of coils required. Thrombin alone is prone for recanalization and may need repeated injections. It could be used as a stopgap arrangement in places where endovascular options are not available and for pseudoaneurysms with failed endovascular access.

\section{Conflict of Interest}

None declared.

\section{References}

1 Shukla AJ, Eid R, Fish L, et al. Contemporary outcomes of intact and ruptured visceral artery aneurysms. J Vasc Surg 2015;61(6):1442-1447

2 Cordova AC, Sumpio BE. Visceral artery aneurysms and pseudoaneurysms-Should they all be managed by endovascular techniques? Ann Vasc Dis 2013;6(4):1-7

3 Cope C, Zeit R. Coagulation of aneurysms by direct percutaneous thrombin injection. Am J Roentgenol 1986;147(2): 383-387

4 Sparrow P, Asquith J, Chalmers N. Cardiovascular and interventional ultrasonic-guided percutaneous injection of pancreatic pseudoaneurysm with thrombin. Case Report 2003;3-6. Doi: 10.6084/m9.figshare.14382065

5 Barbiero G, Battistel M, Susac A, Miotto D. Percutaneous thrombin embolization of a pancreatico-duodenal artery pseudoaneurysm after failing of the endovascular treatment. World J Radiol 2014;6(8):629-635

6 Fankhauser GT, Stone WM, Naidu SG, et al. Mayo Vascular Research Center Consortium. The minimally invasive management of visceral artery aneurysms and pseudoaneurysms. J Vasc Surg 2011;53(4):966-970

7 Francisco LE, Asunción LC, Antonio CA, Ricardo RC, Manuel RP, Caridad MH. Post-traumatic hepatic artery pseudoaneurysm treated with endovascular embolization and thrombin injection. World J Hepatol 2010;2(2):87-90 